\title{
A Face Morphing Detection Concept with a Frequency and a Spatial Domain Feature Space for Images on eMRTD
}

\author{
Tom Neubert \\ Otto-von-Guericke University \\ Magdeburg, Germany \\ tom.neubert@ovgu.de
}

\author{
Christian Kraetzer \\ Otto-von-Guericke University \\ Magdeburg, Germany \\ christian.kraetzer@ovgu.de
}

\author{
Jana Dittmann \\ Otto-von-Guericke University \\ Magdeburg, Germany \\ jana.dittmann@ovgu.de
}

\begin{abstract}
Since the face morphing attack was introduced by Ferrara et al. in 2014, the detection of face morphings has become a wide spread topic in image forensics. By now, the community is very active and has reported diverse detection approaches. So far, the evaluations are mostly performed on images without post-processing. Face images stored within electronic machine readable documents (eMRTD) are $\mathrm{ICAO}^{1}$-passport-scaled to a resolution of $413 \times 531$ and a JPG or JP2fi lesize of 15 kilobytes. This paper introduces a face morphing detection concept with 3 modules (ICAO-aligned preprocessing module, feature extraction module and classification module), tailored for such images on eMRTD. In this work we exemplary design and evaluate two feature spaces for the feature extraction module, a frequency domain and a spatial domain feature space. Our evaluation will compare both feature spaces and is carried out with 66,229 passport-scaled images (64,363 morphed face images and 1,866 authentic face images) which are completly independent from training and include all images provided for the IHMMSEC'19 special session: "Media Forensics - Fake or Real?". Furthermore, we investigate the influence of different morph generation pipelines to the detection accuracies of the concept and we analyse the impact of neutral and smiling genuine faces to the morph detector performance. The evaluation determines a detection rate of $86.0 \%$ for passport-scaled morphed images with a false alarm rate of $4.4 \%$ for genuine images for the spatial domain feature space.
\end{abstract}

\section{KEYWORDS}

Face Morphing; Image Forensics; Morphing Detection; eMRTD

ACM Reference Format:

Tom Neubert, Christian Kraetzer, and Jana Dittmann. 2019. A Face Morphing Detection Concept with a Frequency and a Spatial Domain Feature Space for Images on eMRTD . In ACM Information Hiding and Multimedia Security Workshop (IH\&MMSec '19), fuly 3-5, 2019, Paris, France. ACM, Paris, France, 6 pages. https://doi.org/10.1145/3335203.3335721

\footnotetext{
${ }^{1}$ International Civil Aviation Organization

Permission to make digital or hard copies of all or part of this work for personal or classroom use is granted without fee provided that copies are not made or distributed for profit or commercial advantage and that copies bear this notice and the full citation on thefi rst page. Copyrights for components of this work owned by others than ACM must be honored. Abstracting with credit is permitted. To copy otherwise, or republish, to post on servers or to redistribute to lists, requires prior specific permission and/or a fee. Request permissions from permissions@acm.org.

IH\&MMSec '19, July 3-5, 2019, Paris, France

(C) 2019 Association for Computing Machinery.

ACM ISBN 978-1-4503-6821-6/19/07...\$15.00

https://doi.org/10.1145/3335203.3335721
}

\section{INTRODUCTION}

Face image based authentification scenarios are well-accepted and widespread. In 2014 Ferrara et al. present an identity theft scheme for those scenarios in [8]. They describe an approach allowing two or more persons to pass a face image based authentification scenario with only one artificially weakened Photo-ID template. For the presented attack, a so called face morphing is created, which melts two or more face images of different persons. This morphed face image is used for the document creation performed by a corresponding authority. This Photo-ID document is able to succesfully pass all subjectiv and biometric checks in a boarder control scenario [9]. An example is presented in Figure 1.

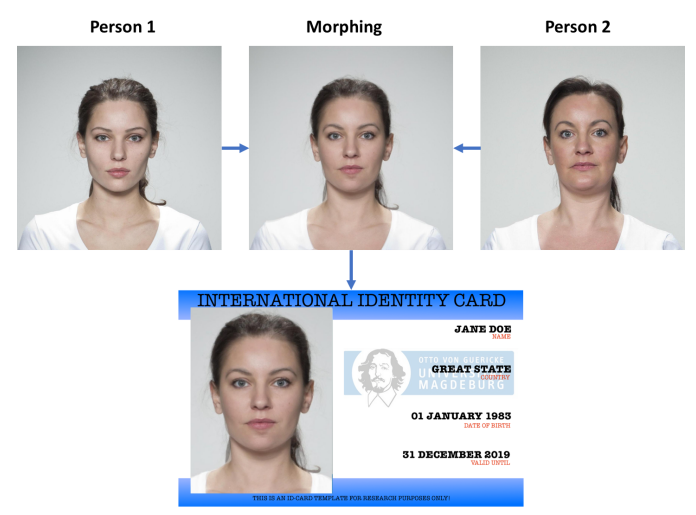

Figure 1: An example of a morphed image on a Photo-ID document template. The images are based on [6]

Because of the simplicity of this attack and the chance for an attacker to easily bypass a boarder control scenario, the forensic community has published different detection approaches such as $[7,14,18,22,25,28]$. A survey about recents works and detection approaches concerning the face morphing attack is given in [20] A challenge for all of these detectors are images with an ICAOcompliant passport-scale which is compatible for electronic machine readable travel documents (eMRTD). It means a resolution of 413x531 pixels and a lossy JPEG (.jpg) / JPEG2000 (.jp2) compression with afi lesize of only $15 \mathrm{~KB}$ [29]. For example, nowdays, a standard photo taken by a Canon EOS $1300 \mathrm{D}$ has a native resolution of 18 megapixels (3456x5184) [3] and a .jpg-filesize of around $5 \mathrm{MB}$. This means a downscaling of $98.84 \%$ andfi lesize reduction of circa $99.7 \%$. So, the post-processing (cropping, scaling and compression) leads to a significant loss of image information. This increases error rates of morphing detectors because expected morphing artifacts (e.g.: 
blending artifacts) could be obscured by this processing operations [24].

Our work addresses the research gap to detect passport-scaled morphed images stored within eMRTD. Our main scentific contribution is a novel face morphing detection concept espacially for passportscaled images on eMRTD. The concept includes 3 modules: an ICAO-aligned pre-processing module, a feature extraction module and a classification module. We exemplary present two different feature spaces for the feature extraction module designed for the challenge to detect passport-scaled images. Thefi rst feature space is designed with frequency domain features as discussed in the information hiding community since the mid-1990s for the detection of the artifacts introduced in digital images by watermarking embedding techniques, see [4]. The second presented feature space is based on spatial domain keypoint features as presented in [14]. The evaluation will compare the performance of the concept with these two different feature spaces and is carried out with 66,229 passport-scaled images (64,363 morphed images and 1866 authentic face images containing neutral and smiling faces) which are completly independent from the training data. Additionally, we compare the detection performance of our concept with .jpg and .jp2files.

\section{STATE OF THE ART}

In this Section, we introduce the morph generation pipelines we have used to create our evaluation data sets. Furthermore, we describe one concept for the feature space module we modify for our purpose to detect passport-scaled morphed images with our detection concept.

\subsection{Morph Generation Pipelines}

For our paper, we use three state-of-the-art morph generation pipelines (MGP) to create three different types of face morphing for our evalatuion dats sets from two related works [19, 24]. As mentioned in [30], all morph pipelines are based on alpha-blending and warping. For our evaluation the alpha-blending parameter is set to 0.5 to build an average between both input images. The warping process is realized with a triangulation which uses 68 facial landmarks, localized by the dlib shape predictor (www.dlib.net). All presented MGP are completly automated processes to create statistically singnificant data sets for training and testing.

- Thefi rst MGP creates a so called complete morph. Here, additionally to the steps above, 20 landmarks on the image borders are added on the image boarders to cover the complete image [21]. Due to this, a complete morph has an average texture and a average geometry of the original faces and is introduced in [19]. This leads to a high biometric quality [19] but also to clearly visible ghosting artifacts as a result of a blending operation, visualized in Figure 2.

- The second morph generation pipeline creates splicing morphs and is introduced in [19] too. The appearance of splicing morphs is more realistic than the appearance of complete morphs, because they are designed to avoid ghosting artifacts. The facial region is cut, warped and blended to a mutual face and stitched back (seamlessly) into one of the input images. This leads to a lower biometric quality and to fewer visible artifacts [19].

- The combined morph is the third MGP used and combines the aforementioned pipelines to avoid shortcomings. The pipeline is introduced in [24] and aligns the original images prior to the warping process to ensure that warping does not lead to a distortion of the face geometry. This MGP has the best visual quality of the three mentioned pipelines as well as a high biometric quality [24].

The 3 MGP are visualized in Figure 2.

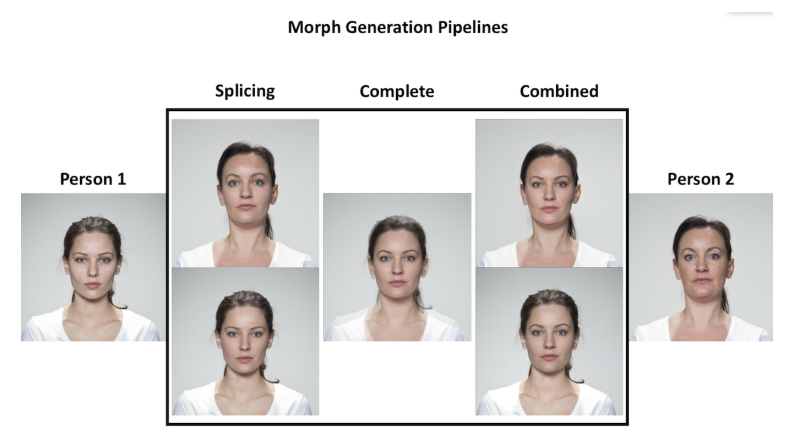

Figure 2: Morph Generation Pipelines (MGP). The images are based on [6]

\subsection{Keypoint Feature Space for Morph Detection}

In [14] a feature space for morph detection is presented, based on the idea that the blending operation in morphing pipelines causes a reduction of face details, especially on images with a resolution with more than 2 megapixels. The reduction of face details is quantified by the number of keypoints and egdes in the face region. Here, the authors assume that the number of keypoints and edges become lower after a blending operation. As pre-processing, prior to feature extraction, the authors segment the face region as a convex hull of the 68 facial landmarks localized with the shape predictor class from the dlib programming library version 19.2 (http://dlib.net/). The authors use several keypoint and edge detectors, because they assume that the impact of blending operation is different for each detector, some of them will react more sensible than others. The following keypoint and edge detectors were used as features for the detector in [14]: SIFT [15], SURF [1], ORB [27], FAST [26], AGAST [17], CannyEdge [2], SobelX and SobelY [13]. These eight features were extracted with functions implemented in the OpenCV programming library version 3.0 with contributions (http://opencv.org). They normalize the number of detected keypoints and edges for every feature with the natural logarithm of the image size (in pixels), because of the different face sizes from different image resolutions. Furthermore, the authors build a lossy JPEG compressed self-reference (quality level: 75\%) and extract the same features. Subsequent they build the ratio between features from the orignal image and the lossy self-reference image. So, it results in a 16 dimensional feature space. 
The work in [24] shows that this detector is not suitable for passportscaled images, because the reduction of thefi lesize and the downscaling also lead to a reduction of keypoints and edges. So, the detector delivers a reasonable high false alarm rate (ca. $42 \%$ ) for genuine images. Furthermore, this rate is complimentary, because the same passport-scaled images but without JPEG compression to $15 \mathrm{kB}$ were involved into training.

In this paper we selected this feature space for our face morphing detection concept and suggest a modification (see Section 3.2.2).

\section{OUR MORPH DETECTION CONCEPT FOR PASSPORT-SCALED IMAGES ON EMRTD}

The chapter introduces our morph detection concept for passportscaled images stored within eMRTD. It includes 3 modules:

- an ICAO-aligned pre-processing module PPM (Section 3.1),

- a feature extraction module FEM (Section 3.2) and

- a classification module $C M$ (Section 3.3).

We introduce in this paper two examplary designed feature spaces for FEM to detect passport-scaled morphs. Thefi rst feature space works in frequency domain $F S_{F r e q}$ and second feature space in spatial domain $F S_{\text {Spat }}$. We will compare the results in our evaluation. The concept is visualized in Figure 3 and the modules will be briefly described in the following Subsections.
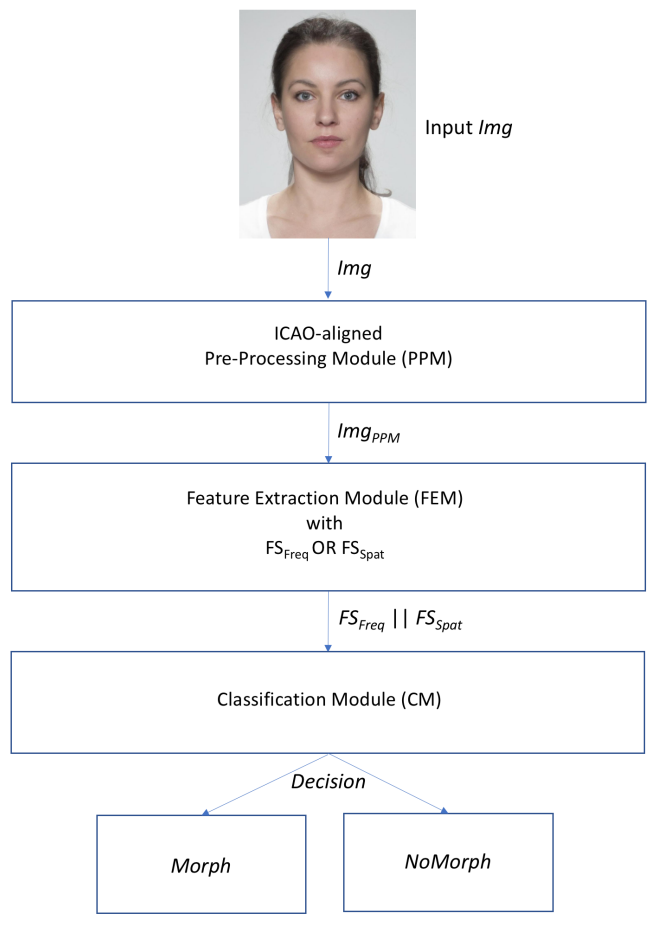

Figure 3: Face Morphing Detection Concept for passportscaled Photo-ID images

\subsection{ICAO-aligned Pre-Processing Module ( $P P M)$}

For our detection concept we only use passport-scaled images because they have completly different characteristics than native images caused by the downscaling to $413 \times 531$ pixels and the compression of the imagefi lesize to $15 \mathrm{kB}$. So, $P P M$ checksfi rst if the input image Img is passport-scaled (1). If the image complies with the requirements from [29], $P P M$ proceeds with the face segmentation (2), if not, the image is converted into a passport-scaled image before the face segmentation. We segment the face region with the help of the 68 facial landmarks from the dlib programming library version 19.2 (http://dlib.net/) and its shape predictor ${ }^{2}$. For the face segmentation, the input image is converted in .pngfile format, because dlib does not support .jp2fi les. With the landmarks we create a bounding box around the face region (region of interest ROI). This region is our new image matrix for processing (3) and it is scaled to a constant resolution of $200 \times 200$ pixels, similarly as proposed in [28]. We choose a constant image size to normalize the face size for the feature extraction module and it results in a normalized feature spaces. The image processing pipeline for the $P P M$ is visualized in Figure 4.

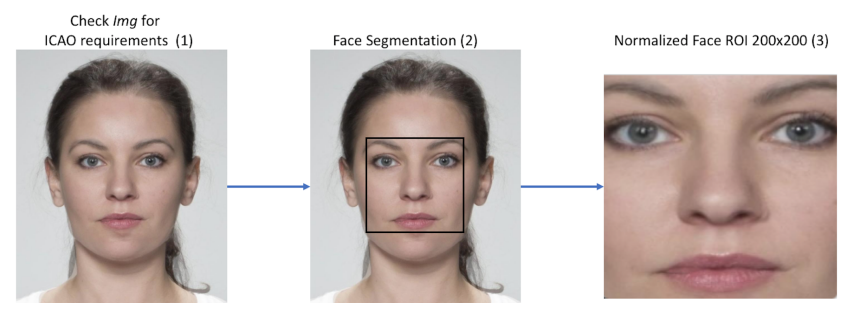

Figure 4: Image Processing in PPM

\subsection{Feature Extraction Module (FEM)}

The input of the FEM is a $200 \mathrm{x} 200$ pixels face image $I m g_{P P M}$ derived from PPM. We have exemplary designed two feature spaces $\left(F S_{F r e q}\right.$ and $F S_{\text {Spat }}$ ) for $F E M$, described in the following subsection. In our evaluation we compare the performance of our detection concept with $F S_{F r e q}$ and $F S_{S p a t}$ in FEM.

3.2.1 Frequency Domain Feature Space $\left(F S_{F r e q}\right)$. The idea to transform images into the frequency domain to detect artifacts is well known. It is used since the mid-1990s for detection of artifacts created by watermarking embedding techniques as discribed in [4] and also for a morph detection approach in [5]. We adapt this idea to create a feature space to detect morphings on passportscaled images. $F S_{F r e q}$ is based on the assumption that the blending operation in the face region during the morphing process causes a reduction of high frequencies (high frequencies contain edge informations) in frequency image domain after a discrete fourier transformation (DFT), even on lossy passport-scaled images. The image processing for $F S_{F r e q}$ starts with the transformation of the image into the frequency domain with a DFT. We perform the DFT with OpenCV version 3.2 and default parametrization as described in their documentation ${ }^{3}$. The DFT of an image $I$ results in a matrix

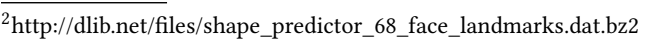

${ }^{3} \mathrm{https}$ //docs.opencv.org/2.4/doc/tutorials/core/discrete_fourier_transform/discrete fourier_transform.html
} 
of complex numbers with a real part Re and a complex (imaginary) part Im. So, we transform the real and complex (imaginary) parts to magnitude $M$ with

$$
M=\sqrt{\operatorname{Re}(D F T(I))^{2}+\operatorname{Im}(D F T(I))^{2}} .
$$

After $M$ is calculated, we re-arrange the quadrants of the magnitude $M$ (1) to set the origin to the image center (see Figure 5). We assume that the blending operations during the morphing process results in a reduction of high frequencies caused by the reduction of edge informations. We try to measure this reduction by seperating $M$ in 25 blocks with $40 \times 40$ pixels (2). In this blocks we calculate the mean magnitude values as features (3). So, $F S_{F r e q}$ results in a 25 dimensional vector.

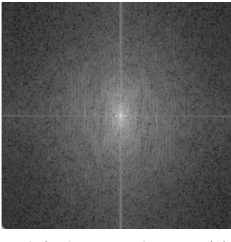

Shifted Magnitude Image (1)

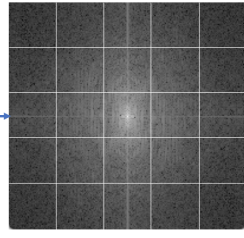

Seperate 25 Blocks (2)

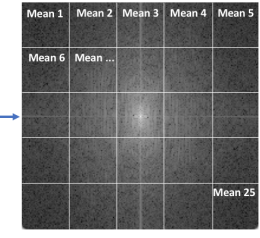

Feature Extraction (3)
Figure 5: Image Processing for $F S_{F r e q}$

3.2.2 Spatial Domain Feature Space $\left(F S_{S p a t}\right)$. As mentioned before, $F S_{\text {Spat }}$ is based on the work of Kraetzer et al. in [14]. The approach is thoroughly described in Section 2.2. Basically, we primarily modify the feature normalization and the feature selection compared to [14]. The number of detected keypoint and edge features from 8 detectors (SIFT, SURF, ORB, FAST, AGAST, CannyEdge, SobelX and SobelY, see Section 2.2) are extracted on the normalized face image derived from $P P M$. Hence, no more feature normalization is required and we extract a 8 dimensional feature vector.

\subsection{Classification Module ( $C M)$}

$C M$ is based on feature vectors generated by FEM to train a statistical model. We train overall 4 statistical models in this work, two for each feature space $\left(F S_{F r e q}\right.$ and $\left.F S_{S p a t}\right)$ with each one for the allowed image datatypes (.jpg and .jp2) [29]. Hence, we get 4 the statistical models $M_{F S_{F r e q} J P G}, M_{F S_{F r e q} J P 2}, M_{F S_{S p a t} J P G}$ and $M_{F S_{S p a t} J P 2}$, were the models with .jpgfi les $\left(M_{F S_{F r e q} J P G}\right.$, $M_{F S_{S p a t} J P G}$ ) were used to classify only .jpg test datasets and those with.jp2fi les $\left(M_{F S_{F r e q} J P 2}, M_{F S_{S p a t} J P 2}\right)$ to classify .jp2 test datasets. All models have 2 classes (Morph and NoMorph). The training data consists of self acquired authentic images (NoMorph) and morphs which are created based on these authentic images. We use images with neutral face expression to train the statistical models, a detailed describtion of the training data is given in Section 4.1. The statistical models are trained with WEKA 3.8 [11] and its SMO classifier. $C M$ decides whether an image is morphed or not.

\section{EVALUATION SETUP}

Our face morphing detection concept is evaluated with two different feature spaces $F S_{\text {Freq }}$ and $F S_{\text {Spat }}$ (see Section 3.2). Therefore, we create statistical models with self-acquired training data (Section
4.1). We evaluate our concept with both feature spaces and with independent free available test data and define our test goals in Section 4.2 .

\subsection{Training Data}

Our training data includes 12,500 images (4,234 NoMorphs; 8,266 Morphs) based on 90 different persons for all trained models. The 90 subjects have a neutral face expression and were acquired mostly with 3 different cameras and two instances of each camera model including 3 different resolutions and 3 different ISO-values per camera, based on the ICAO-requirements (see Table 1).

Table 1: Used cameras and acquisition parameters for the training data. * Resolution before the passport-scale.

\begin{tabular}{lcc}
\hline \multicolumn{1}{c}{ Camera } & Resolution* & ISO-value \\
\hline 2 * Canon EOS 1200D & $3456 \times 5184$ & $100,400,800$ \\
Lens: EF28-1005 f/4-5.6 & $2304 \times 3456$ & $100,400,800$ \\
& $1728 \times 2592$ & $100,400,800$ \\
\hline $2 *$ Nikon D3300 & $4000 \times 6000$ & $100,400,800$ \\
Lens: AF-S 50mm f/1.8G & $3000 \times 4496$ & $100,400,800$ \\
& $2000 \times 2992$ & $100,400,800$ \\
\hline \multirow{2}{*}{ * Nikon Coolpix A100 } & $3834 \times 5152$ & $100,400,800$ \\
& $1703 \times 2272$ & $100,400,800$ \\
& $1200 \times 1600$ & $100,400,800$ \\
\hline
\end{tabular}

Morphings in our training dataset are based on those 90 persons and are created with the three used morph generation pipelines presented in Section 2.1. The dataset includes 8,266 morphs in which the three generation pipelines are nearly equal distributed $(2,995$ complete morphs, 2,637 splicing morphs, 2,634 combined morphs). These attack types represent realistic attacks with a nearly faultless visual quality of the face region. As mentioned before, all 12,500 samples in the training dataset are post-processed with the passportscale and are stored in .jpg and .jp2fi le format. So, our four statistical classification models are all build from 12,500 feature vectors resulting from one of the two detection approaches and include only vectors either from .jpg or .jp2fi les as mentioned in Section 3.3.

\subsection{Test Data and Goals}

Our morph detection concept is evaluated with test datasets which are not included into training. Therefore, we create multiple test datasets with the 3 different morph generation pipelines and genuine images (neutral and smiling expression, NoMorphs). We are aware that smiling faces are not ICAO-compliant, but we evaluate them to analyse the robustness of our detection concept for genuine faces. Furthermore, we use the test datasets provided at IHMMSEC2019 Special Session: Media Forensics - Fake or Real? All test samples in all test datasets are created in bothfi le formats .jpg and .jp2 (except the provided morph test dataset from IHMMSEC). The datasets are listed in Table 2. Hence, our tests are carried out with 66,229 passport-scaled images (64,363 morphed images and 1,866 authentic face images).

We define 5 test goals to evaluate our detection concept with both presented feature spaces.

- G1: Determine the detection performance of the detection concept with $F S_{F r e q}$ and $F S_{S p a t}$. 
- G2: Determine the impact of different morph generation pipelines (complete-, splicing-, combined morph) for the detection concept with $F S_{\text {Freq }}$ and $F S_{S p a t}$.

- G3: Determine the impact of a neutral and smiling face expression for genuine images to the false alarms of the detection concept with $F S_{\text {Freq }}$ and $F S_{\text {Spat }}$.

- G4: Determine the impact of thefi le format .jpg and .jp2 for the detection concept with $F S_{F r e q}$ and $F S_{S p a t}$.

- G5: Compare the performance of the detection concept with $F S_{\text {Freq }}$ vs. $F S_{\text {Spat }}$ (See Section 5.3).

The detection performance is measured with the detection accuracy $A C C$, which is presented in chapter 5 .

Table 2: Overview of used test datasets for evaluation of morph detection approaches. ${ }^{*}$ Resolution before the passport-scale. ${ }^{* *}$ Morphs are based on this databases

\begin{tabular}{lcccc}
\hline \multicolumn{1}{c}{ Test Dataset } & Format & $\begin{array}{c}\text { Reso- } \\
\text { lution }\end{array}$ & Files & $\begin{array}{c}\text { Data } \\
\text { Ref }\end{array}$ \\
\hline CombinedMorphsIHMMSEC19 & jpg & $1350 \times 1350$ & 2,175 & {$[10]$} \\
\hline CompleteMorphsLondon & jpg | jp2 & $1350 \times 1350$ & $2 * 5,050$ & {$[6]^{* *}$} \\
\hline SplicingMorphsLondon & jpg | jp2 & $1350 \times 1350$ & $2 * 9,352$ & {$[6]^{* *}$} \\
\hline CombinedMorphsLondon & jpg | jp2 & $1350 \times 1350$ & $2 * 10,100$ & {$[6]^{* *}$} \\
\hline CompleteMorphsutrecht & jpg | jp2 & $900 \times 1200$ & $2 * 1,326$ & {$[12]^{* *}$} \\
\hline SplicingMorphsutrecht & jpg | jp2 & $900 \times 1200$ & $2 * 2,614$ & {$[12]^{* *}$} \\
\hline CombinedMorphsutrecht & jpg | jp2 & $900 \times 1200$ & $2 * 2,652$ & {$[12]^{* *}$} \\
\hline NoMorphs-neutral IHMMSEC19 & jpg | jp2 & $1350 \times 1350$ & $2 * 102$ & {$[10]$} \\
\hline NoMorphs-smilingIHMMSEC19 & jpg | jp2 & $1350 \times 1350$ & $2 * 102$ & {$[10]$} \\
\hline NoMorphs-neutralutrecht & jpg | jp2 & $900 \times 1200$ & $2 * 74$ & {$[12]$} \\
\hline NoMorphs-smilingUtrecht & jpg | jp2 & $900 \times 1200$ & $2 * 58$ & {$[12]$} \\
\hline NoMorphs-neutralcFD & jpg | jp2 & $2444 \times 1718$ & $2 * 597$ & {$[16]$} \\
\hline
\end{tabular}

\section{EVALUATION RESULTS}

This section presents the evaluation results for our test goals (G1 to G5, see Section 4.2). We show the results for G1 to $\mathbf{G 4}$ of the concept with $F S_{\text {Freq }}$ in Section 5.1 and with $F S_{\text {Spat }}$ in 5.2. The results for test goal G5 are presented in Chapter 5.3. We simply measure the detection performance of the concept with the accuracy of the detectors with $A C C=\frac{C C I}{I N} * 100$ where $C C I$ is the number of correct classified instances in the test dataset and $I N$ is the total number of instances in dataset.

\subsection{Evaluation Results of Detection with $F S_{\text {Freq }}$}

The results of our detection concept with $F S_{\text {Freq }}$ are listed in Table 3. It shows that the concept is able to detect $75.2 \%$ (48,427 samples out of 64,363) of passport-scaled morphed images correctly (G1). Furthermore, it has an accuracy of $95 \%$ for genuine images (5\% false alarms), where especially the images from the UtrechtDB [12] trigger most false alarms. Indeed, these error rate is too high for a real world application, but it can be reduced by a multi-level-detection process, as described in [23]. The morph generation pipelines have a not negligible influence to the detection performance of the concept with $F S_{\text {Freq }}$, especially the performance for the splicing morph varies in comparison to the other pipelines (G2). Furthermore, the results indicate that it has no influence on the detecton performance
Table 3: Detection results of the concept with $F S_{F r e q}$.

\begin{tabular}{|c|c|c|}
\hline Morph Test Dataset & ACC JPG & ACC JP2 \\
\hline CombinedMorphSIHMMSEC19 & 80.9 & - \\
\hline CompleteMorphsLondon & 78.1 & 70.7 \\
\hline SplicingMorphSLondon & 65.1 & 62.5 \\
\hline CombinedMorphsLondon & 75.5 & 68.1 \\
\hline CompleteMorphsutrecht & 95.9 & 93.7 \\
\hline SplicingMorphsutrecht & 97.7 & 99.4 \\
\hline CombinedMorphsutrecht & 96.0 & 96.0 \\
\hline \multirow{2}{*}{ Total Morphs } & 77.5 & 72.8 \\
\hline & \multicolumn{2}{|c|}{75.2} \\
\hline NoMorphs-neutralıнммSEC19 & 100.0 & 98.0 \\
\hline NoMorphs-smilingIHMMSEC19 & 99.0 & 99.0 \\
\hline NoMorphs-neutralutrecht & 70.3 & 68.0 \\
\hline NoMorphs-smilingutrecht & 70.7 & 68.5 \\
\hline NoMorphs-neutralcFD & 100.0 & 98.2 \\
\hline \multirow{2}{*}{ Total NoMorphs } & 95.7 & 94.2 \\
\hline & \multicolumn{2}{|c|}{95.0} \\
\hline \multirow{2}{*}{ Total } & 78.5 & 73.3 \\
\hline & \multicolumn{2}{|c|}{75.8} \\
\hline
\end{tabular}

if a person is smiling or not (G3). The detection accuracy for .jpg files (78.5\%) is significant higher than for .jp2 images (73.3\%) (G4).

\subsection{Evaluation Results of Detection with $F S_{S p a t}$}

The detection concept with $F S_{\text {Spat }}$ is able to detect $86.0 \%$ (55,373 out of 64,363 samples) morphed passport-scaled images correctly and triggers a false alarm rate of $4.4 \%$ for genuine images (G1). The results for all test datasets are shown in Table 4 . We can assert, that the detection accuracy for the morph generation pipeline splicing morph also varies for the concept with $F S_{S p a t}(\mathrm{G} 2)$. Besides, the concept with $F S_{S p a t}$ is less robust for smiling faces as with $F S_{F r e q}$, we notice a varying detection accuracy for these test samples (G3). The detection accuracy for .jpgfi les $(86.7 \%)$ over all datasets is higher than for .jp2fi les (85.9\%) (G4).

\section{3 $F S_{\text {Freq }}$ vs. $F S_{\text {Spat }}$ (G5)}

In this section, we compare the detection accuracies of concept with the both exemplary designed feature spaces. Thefi rst view, shows better results for the detection concept with $F S_{\text {Spat }}$ because it classifies more morphed $(+14.3 \%)$ and more genuine $(+0.6 \%)$ samples correct. We assume this depends on the training data. The dimensionality of the feature space of $F S_{\text {Freq }}$ is higher than the dimensionality of the feature space of $F S_{S p a t}$ and as a result it needs more training samples to deliver more accurate results. But, the detection concept with $F S_{F r e q}$ shows more robust results for neutral and smiling faces in our evaluation. Furthermore, $F S_{\text {Freq }}$ has more accurate results for the "IHMMSEC'19 special session: fake or real" test data with $80.9 \%$ of detected morphs.

\section{CONCLUSION}

This paper introduces a detection concept for face morphings on passport-scaled images on eMRTD. The concept is evaluated with two different feature spaces (FS). The concept is able to detect morphed images with an accuracies of $86.0 \%$ by a false alarm rate 
Table 4: Detection results of the concept with $F S_{S p a t}$.

\begin{tabular}{|c|c|c|}
\hline Morph Test Dataset & ACC JPG & ACC JP2 \\
\hline CombinedMorphSIHMMSEC19 & 77.7 & - \\
\hline CompleteMorphSLondon & 86.5 & 81.3 \\
\hline SplicingMorphsLondon & 81.2 & 81.7 \\
\hline CombinedMorphsLondon & 86.4 & 84.1 \\
\hline CompleteMorphsutrecht & 95.9 & 95.0 \\
\hline SplicingMorphsutrecht & 97.7 & 99.5 \\
\hline CombinedMorphsutrecht & 95.5 & 95.6 \\
\hline \multirow{2}{*}{ Total Morphs } & 86.4 & 85.7 \\
\hline & \multicolumn{2}{|c|}{86.0} \\
\hline NoMorphs-neutralıнммSEC19 & 99.0 & 98.0 \\
\hline NoMorphs-smilingIHMMSEC19 & 99.0 & 98.0 \\
\hline NoMorphs-neutralutrecht & 89.2 & 60.0 \\
\hline NoMorphs-smiling Utrecht & 79.3 & 63.8 \\
\hline NoMorphs-neutralCFD & 100.0 & 99.8 \\
\hline \multirow{2}{*}{ Total NoMorphs } & 97.0 & 94.1 \\
\hline & \multicolumn{2}{|c|}{95.6} \\
\hline \multirow{2}{*}{ Total } & 86.7 & 85.9 \\
\hline & \multicolumn{2}{|c|}{86.3} \\
\hline
\end{tabular}

(FAR) of $4.4 \%$ with a spatial domain FS and with an accuracy of $75.2 \%$ by a FAR of $5 \%$ with a frequency domain FS. The used morph generation pipelines have a not negligible influence to the detection performance of the concept. The frequency domain FS delivers a lower error rate for genuine image with smiling face expression than the spatial domain FS. The concept has a higher detection accuracy for .jpgfi les than for .jp2fi les with both FS. In future work, it would be helpful to create bigger training datasets with an extended variety, this sould increase the detection accuracy. It would be interesting to see how the concept works with other FS and to analyse the influence of anti-forensics to the concept.

\section{ACKNOWLEDGMENT}

The work in this paper has been funded in part by the German Federal Ministry of Education and Research (BMBF) through the research programme ANANAS under contract no. FKZ: 16KIS0509K.

\section{REFERENCES}

[1] Herbert Bay, Andreas Ess, Tinne Tuytelaars, and Luc Van Gool. 2008. Speeded-Up Robust Features (SURF). Comput. Vis. Image Underst. 110, 3 (June 2008), 346-359. https://doi.org/10.1016/j.cviu.2007.09.014

[2] J Canny. 1986. A Computational Approach to Edge Detection. IEEE Trans. Pattern Anal. Mach. Intell. 8, 6 (June 1986), 679-698. https://doi.org/10.1109/TPAMI.1986. 4767851

[3] Canon-Europe. 2018. Canon EOS 1300D Specifications. (2018) https://www.canon-europe.com/for_home/product_finder/cameras/digital_slr/ eos_1300d/specification.aspx

[4] Ingemar Cox, Matthew L. Miller, and Jeffery A. Bloom. 2002. Digital watermarking. Morgan Kaufmann Publishers Inc. ; ISBN: 1-55860-714-5 (2002).

[5] Luca Debiasi, Ulrich Scherhag, Christian Rathgeb, Andreas Uhl, and Christoph Busch. 2018. PRNU-based Detection of Morphed Face Images, In 6th International Workshop on Biometrics and Forensics, IWBF 2018, Sassari, Italy, June 6-7, 2018. Digital Forensics and Watermarking.

[6] Lisa M. DeBruine and Benedict C. Jones. 2017. Face Research Lab London Set figshare doi: 10.6084/m9.figshare.5047666 (2017). https://figshare.com/articles/ Face_Research_Lab_London_Set/5047666/3

[7] David J. Robertson et al. 2018. Detecting morphed passport photos: a training and individual differences approach. Cognitive Research: Principles and Implications 3:27 (2018).
[8] Matteo Ferrara, Annalisa Franco, and Davide Maltoni. 2014. The magic passport. In $I F C B$. IEEE, 1-7.

[9] Matteo Ferrara, Annalisa Franco, and Davide Maltoni. 2016. On the Effects of Image Alterations on Face Recognition Accuracy. In Face Recognition Across the Imaging Spectrum. Springer, 195-222.

[10] AMSL Research Group. [n. d.]. 2019 AMSL Face Morph Image Data Set. ([n. d.]). https://omen.cs.uni-magdeburg.de/disclaimer/index.php

[11] M. Hall. 2009. The WEKA data mining software: An update. In SIGKDD Explorations (2009).

[12] P. Hancock. [n. d.]. Psychological image collection at stirling (pics) - 2d face sets - utrecht ecvp, http://pics.psych.stir.ac.uk/ last accessed: 2017/07/14. ([n. d.])

[13] N. Kanopoulos, N. Vasanthavada, and R.L. Baker. 1988. Design of an image edge detectionfi lter using the Sobel operator. IEEE Journal of Solid-State Circuits (Volume: 23, Issue: 2, Apr 1988) DOI: 10.1109/4.996 (1988).

[14] Christian Kraetzer, Andrey Makrushin, Tom Neubert, Mario Hildebrandt, and Jana Dittmann. 2017. Modeling Attacks on Photo-ID Documents and Applying Media Forensics for the Detection of Facial Morphing. In Proceedings of the 5th ACM Workshop on Information Hiding and Multimedia Security (IHMMSec '17). ACM, New York, NY, USA, 21-32. https://doi.org/10.1145/3082031.3083244

[15] David G. Lowe. 1999. Object Recognition from Local Scale-Invariant Features. In Proceedings of the International Conference on Computer Vision-Volume 2 - Volume 2 (ICCV '99). IEEE Computer Society, Washington, DC, USA.

[16] Debbie S. Ma, Joshua Correll, and Bernd Wittenbrink. 2015. The Chicago face database: A free stimulus set of faces and norming data. Behavior Research Methods 47, 4 (01 Dec 2015), 1122-1135. https://doi.org/10.3758/s13428-014-0532-5

[17] Elmar Mair, Gregory D. Hager, Darius Burschka, Michael Suppa, and Gerhard Hirzinger. 2010. Adaptive and Generic Corner Detection Based on the Accelerated Segment Test. In Proceedings of the 11th European Conference on Computer Vision: Part II (ECCV'10). Springer-Verlag, Berlin, Heidelberg, 183-196. http://dl.acm. org/citation.cfm?id=1888028.1888043

[18] Andrey Makrushin, Christian Kraetzer, Tom Neubert, and Jana Dittmann. 2018. Generalized Benford's Law for Blind Detection of Morphed Face Images. In IHMMSec '18: 6th ACM Workshop on Information Hiding and Multimedia Security, June 20-22, 2018, Innsbruck, Austria. ACM, Innsbruck, Austria, 6 pages. (2018).

[19] Andrey Makrushin, Tom Neubert, and Jana Dittmann. 2017. Automatic Generation and Detection of Visually Faultless Facial Morphs. In Proceedings of the 12th International foint Conference on Computer Vision, Imaging and Computer Graphics Theory and Applications - Volume 6: VISAPP, (VISIGRAPP 2017).

[20] Andrey Makrushin and Andreas Wolf. [n. d.]. An Overview of Recent Advances in Assessing and Mitigating the Face Morphing Attack. In Proc.EUSIPCO'18, pp1022-1026 ([n. d.]).

[21] S. Mallick. [n. d.]. Face morph using opencv - c++/ python. ([n. d.]). http: //www.learnopencv.com/face-morph-using-opencv-cpp-python/

[22] Tom Neubert. 2017. Face Morphing Detection: An Approach Based on Image Degradation Analysis. In Digital Forensics and Watermarking, Christian Kraetzer, Yun-Qing Shi, Jana Dittmann, and Hyoung Joong Kim (Eds.). Springer International Publishing, UK, pp 93-106.

[23] Tom Neubert, Christian Kraetzer, and Jana Dittmann. [n. d.]. Reducing the False Alarm Rate for Face Morph Detection by a Morph Pipeline Footprint Detector. In EUSIPCO 2018: 26th European Signal Processing Conference : Rome, Italy, Septemberr 3-7, 2018 - Rome, insges. 5 S. ; [Konferenz: 6th European Signal Processing Conference, EUSIPCO 2018, Rome, Italy, Septemberr 3-7, 2018]. 1002-1006. https://doi.org/10.23919/EUSIPCO.2018.8553067

[24] Tom Neubert, Andrey Makrushin, Mario Hildebrandt, Christian Kraetzer, and Jana Dittmann. 2018. Extended StirTrace Benchmarking of Biometric and Forensic Qualities of Morphed Face Images. IET Biometrics 2018 (2018).

[25] Ramachandra Raghavendra, Kiran B. Raja, S. Venkatesh, and Christoph Busch. 2017. Transferable Deep-CNN features for detecting digital and print-scanned morphed face images. In Proceedings of 30th International Conference on Computer Vision and Pattern Recognition Workshop (CVPRW 2017), Honolulu, Hawaii, fuly 21-26, 2017. 10-18.

[26] Edward Rosten and Tom Drummond. 2005. Fusing Points and Lines for High Performance Tracking. In Proceedings of the Tenth IEEE International Conference on Computer Vision - Volume 2 (ICCV '05). IEEE Computer Society, Washington, DC, USA, 1508-1515. https://doi.org/10.1109/ICCV.2005.104

[27] Ethan Rublee, Vincent Rabaud, Kurt Konolige, and Gary Bradski. 2011. ORB: An Efficient Alternative to SIFT or SURF. In Proceedings of the 2011 International Conference on Computer Vision (ICCV'11). IEEE Computer Society, Washington, DC, USA, 2564-2571. https://doi.org/10.1109/ICCV.2011.6126544

[28] Clemens Seibold, Wojciech Samek, Anna Hilsmann, and Peter Eisert. 2017. Detection of Face Morphing Attacks by Deep Learning. In Digital Forensics and Watermarking, Christian Kraetzer, Yun-Qing Shi, Jana Dittmann, and Hyoung Joong Kim (Eds.). Springer International Publishing, UK, pp 107-120.

[29] ISO/IEC JTC1 SC17 WG3. 2018. ICAO Technical Report Portrait Quality (Reference Facial Images for MRTD). Version: 1.0. (2018). https://www.icao.int/ Security/FAL/TRIP/Documents/TR\%20-\%20Portrait\%20Quality\%20v1.0.pdf

[30] G. Wolberg. 1998. Image morphing: a survey. In Visual Computer 14(8), pp. 360-372. (1998) 